\title{
The effectiveness of intravesical Botox in idiopathic and non-idiopathic detrusor over activity
}

Accepted: $6 / 11 / 2010$

\begin{tabular}{ccc}
\hline Soran Rafaat Ismail* & Bahzad Koye** \\
\hline \multicolumn{3}{c}{ Abstract }
\end{tabular}

Background and objectives: We have evaluated and compared the efficacy of Botox® in the treatment of bladder over activity in Idiopathic detrusor over activity (IDO) and Neuropathic detrusor over activity (NDO) in a tertiary centre. We hypothesised that the outcome of this less invasive treatment would be more promising in patients with IDO than those with NDO.

Methods: A total of 229 patients were reviewed with overactive bladder proven by urodynamic studies over the last 2 years. Of these, 174 patients received Botox® injection. 132 (75\%) patients had IDO and $42(25 \%)$ patients had NDO. 200 IU of Botox® was injected per patient in the IDO group and $300 \mathrm{IU}$ in the neurogenic group.

Results: Eighty one of $118(68.7 \%)$ known patients with IDO reported being dry after receiving the Botox® compared to $20 / 39(51.3 \%)$ patients with NDO. Whereas, 88/118 $(68.7 \%)$ patients with IDO reported significant satisfaction compared to $25 / 39(64.1 \%)$ patients with NDO.

Conclusion: Botox® is an effective, minimally invasive treatment for drug refractory IDO + NDO. In comparison, patient reported satisfaction and dryness with the procedure was statistically the same. However, the IDO group responded slightly better to treatment. The average mean effect was the same in both groups.

Key words: Botox, Idiopathic detrusor over activity (IDO), Neuropathic detrusor over activity (NDO).

\section{Introduction}

Treatments of overactive bladder (OAB) include conservative measures such as lifestyle change, bladder training or pelvic rehabilitation, and local oestrogen if there is female genital atrophy. Oral anticholinergic agents with or without clean intermittent self catheterisation (CISC) are a second line therapy, with documented suppressive effect on symptoms in about $70 \%$ of cases 1,2. The limiting factor for the use of these medications in the long term are side effects, like dry mouth, constipation, headache and nausea ${ }^{3-5}$. If the patient is unable to tolerate these drugs or failing to respond, they may live with their symptoms, or undergo more invasive treatments such as sacral neuromodulation ${ }^{6}$ or bladder augmentation ${ }^{7}$. Treatment with botulinum toxin (BTX) is an intermediate treatment options that fill the therapeutic gap between oral medication and reconstructive bladder surgery. Although other treatments have been proposed to fill this gap for example, instilling intravesical neurotoxins (capsaicin and resiniferatoxin), they remained unlicensed because of the conflicting results of the efficacy in placebocontrolled trails ${ }^{8-10}$. Administration of BTX directly into the bladder wall is a new minimally invasive treatment concept, which has been increasingly researched and applied. A significant relief of symptoms in patients with NDO and IDO have been documented ${ }^{11-15}$. Comparable urodynamic

*Neuro-Urology Department, Royal National Orthopa dic Hospital- Stanmore, UK.

**Urology Department, the James Cook University Hospital, UK 
and symptomatic improvements have been demonstrated between patients with IDO and those with NDO ${ }^{16}$. Botulinum toxin (BTX) is a neurotoxin protein produced by the Gram positive, rod shaped anaerobic bacteria Clostridium botulinum ${ }^{17}$. It is the most poisonous naturally occurring toxin known to humans . BTX-A is manufactured in the USA by Allergan Inc. for both therapeutic and cosmetic use and it is known as Botox®. Whereas in the UK and Europe it is developed and manufactured in Ireland and is known as Dysport ${ }^{\circ}$. BTX-A is more potent and has a longer duration of action than type B. BTX-A is known to be a selective inhibitor of Ach release. The mechanism of action, at least on the motor side, is ultimately by interrupting the fusion of vesicles containing Ach to the plasma membrane of the peripheral cholinergic terminals. Thus, it prevents the release of Ach at the neuromuscular junction, resulting in flaccid paralysis of the muscle ${ }^{18}$.

The aim of this study is to evaluate and compare the efficacy of BTX-A injections in the treatment of detrusor over activity in IDO and in NDO. We hypothesized that the outcome of this less invasive treatment would be more promising in patients with IDO than those with NDO.

\section{Methods}

Ethical approval was not required as this is a retrospective study. Information and advice was sought from the local statistician to establish a statistically significant cohort. The Fisher Exact Test was used to analyze the data as opposed to the Chi-square test as it was more accurate for this particular study .This study was conducted from the results of one urology department at the University College London Hospitals, a major tertiary referral centre. Between the years 2006 and 2009, 229 patients were recommended to have Botulinum toxin $A$ for treatment of $\mathrm{UI}$ as part of their clinical management of which 174 went on to have the procedure performed. All operations were performed by the same two consultant urologists for all patients. The technique of BTX-A injection is standardized and performed consistently by these two surgeons. All these patients had cystoscopy and intradetrusor injection with BTXA. Botox $®$ is the preparation used at this centre and was given to 174 patients in doses ranging from 100-300 units, which was diluted in $\mathrm{NaCl} 0.9 \%$ in a rate of $10 \mathrm{IU}$ Botox® to $1 \mathrm{ml}$ of $\mathrm{NaCl} 0.9 \%$. Cystoscopy was performed and bladder mucosa was examined, to exclude any abnormalities, prior to the injection of BTX. Twenty to thirty sites were injected covering the maximum bladder surface avoiding the trigonal and bladder neck region, and the blood vessels. All of the patients were admitted and discharged on the same day. Patients were advised to continue their anticholinergic medication for 2 weeks after the BTX-A injections and then to withdraw the medications completely. There is no variation in the surgical technique compared to other studies. Of the 174 patients that received the BTX treatment 132 were urodynamically proven to have IDO and 42 patients were urodynamically proven to have NDO, and all were refractory to anticholinergics. Age range of the patients with IDO was 2088 years (mean 54.21) and for the NDO group ranged from 7-74 years (mean 40.66).Those patients who had Botox® treatment for causes other than IDO and NDO were excluded from this study. A further exclusion was those patients being treated with BTX A at other centers by the same two surgeons.

\section{Results}

The notes for 229 patients were reviewed that had urodynamically proven overactive, of these 174 received BTX-A treatment. The age group of the study group was between 7 years old and 88 years old with a mean age of 50.94 years. There were 54 $(31.03 \%)$ men and 120 (68.97\%) women. The IDO group consisted of 95 (71.96\%) female and $37(28.04 \%)$ male patients with an age range of 20 years old to 88 years old (mean age 54.21 years). The Botox® injection procedure was performed under 
local anaesthetic in 40 patients and the remaining 92 had their procedure undertaken by general anaesthetic. The majority of patients received $200 \mathrm{IU}$ of Botox ${ }^{\circledR}$, numbering 124 patients, 3 patients received 100 $\mathrm{IU}, 2$ patients received $240 \mathrm{IU}$ and 3 patients received $300 \mathrm{IU}$. Eighty one (68.7\%) out of 118 patients reported being dry post Botox $\circledast$ procedure. This equated to 19 men and 62 women. Thirty seven patients were not dry (31.3\%) and 18 of them under went further treatment. Of the 37 patients 13 were men 24 were women. Eighty eight patients $(74.6 \%)$ stated they were satisfied with their treatment outcome, 20 men and 68 women. Thirty patients $(25.4 \%)$ were not satisfied, 12 men and 18 women. The remaining 14 patients are unknown. For those patients dry post Botox ${ }^{\circledR}$ injection (81) the incidence of urinary retention was $9.8 \%$ (8 patients). This occurred in 4 male and 4 female patients. Intermittent Self Catheterization (ISC) was performed in 6 (6 out of $81=7.4 \%$ ) out of the 8 patients in urinary retention post Botox ${ }^{\circledR}$ procedure which equated to 2 males and 4 females. Of the remaining 2 patients, 1 male $1 \mathrm{fe}-$ male they were managed with an indwelling catheter. Eight patients out of the 132 patients reported developing a Urinary Tract Infection (UTI) (6.06\%) post procedure and 28 patients had pre-existing Urinary Tract Infections. Patients reported the duration of action of Botox $®$ as 9-12 months with 2 patients reporting effectiveness of less than 2 month and 1 patient reporting effectiveness at 18 months. The average duration of effectiveness \pm standard deviation was 10.15 ( \pm 3.39) month. The mean duration of action between the second and third Botox $®$ injection \pm the standard deviation was $8.43( \pm 2.82)$. Of the 132 patients, 54 patients went on to receive a second dose of Botox $®, 21$ patients received 3 doses and only 1 patient received 4 doses. 2 patients failed to achieve a therapeutic outcome after the first dose. In group NDO Twenty (51.3\%) out of 39 patients reported being dry post Botox ${ }^{\circledR}$ procedure. This equated to 7 men and 13 women. Nineteen patients were not dry $(48.7 \%)$ and 8 of them went for further treatment. Of the 19 patients 9 were men 10 were women. The remaining 3 patients are unknown. Twenty five patients $(64.1 \%)$ stated they were satisfied with their treatment outcome, (11 men and 14 women). Fourteen patients $(35.9 \%)$ were not satisfied, 5 men and 9 women. The remaining 3 patients are unknown. Urinary retention was reported in 2 patients out of 4 patients that were voiding independently prior to Botox® $(50 \%)$. One patient was managed with ISC and the other with an SPC. Ten patients performed ISC pre Botox $\AA$ of which 9 continue to do so post Botox $®$ and the remaining one patient converted to a SPC. The reported incidence of Urinary Tract Infection was 2 out of 42 patients post Botox® treatment (4.7\%). Twelve patients out of 42 reported recurrent UTI. Patients reported the duration of action of Botox® as 9-12 months with 4 patients reporting effectiveness of more than 12 month. The average duration of effectiveness \pm standard deviation was 10.76 ( \pm 3.63) month. The mean duration of action between the second and third Botox $\AA$ injection \pm the standard deviation was 7.8 ( \pm 2.04). Out of the 42 patients that received Botox $®$ treatment, 14 then received a second dose, 6 patients had 3 doses and 1 had 4 doses. Fourteen patients went onto have further urological procedures undertaken. Of which 8 were from the nonresponder group ${ }^{19}$ and 4 were from the responder group ${ }^{20}$. 


\section{Discussion}

Botulinum toxin is slowly emerging as a suitable alternative and a possible longterm treatment option for managing patients with $\mathrm{OAB}{ }^{9}$. In recent years, BTX-A has increasingly been used to manage conditions characterised by DO where the patient is unwilling to have or unsuitable for a surgical treatment. To date, numerous studies have been published on the results of BTX-A intravesical injections to treat both. Data have reported the long-term efficacy and tolerability of the neurotoxin after repeated injections in patients affected by NDO. $3,5,13,21$ The number of patients suffering from IDO is 132 which is higher than and not equal to the number of patients with NDO at 42. However, statistically it is still significant and comparable as the previous studies were based on less number of patients. ${ }^{16,19}$ In addition, to keep consistency with our procedure and avoid mistakes we only included patients from the same centre and who had their treatments given by the same two surgeons. This has resulted in an unequal number, but, we have fewer variables for the results. There were $120(68.97 \%)$ women and 54 $(31.03 \%)$ men. This is a reflection of the demographics of the disease (female: male is 760: 597 million in 2008). ${ }^{15}$ The demographics are representative of the presentation of this type of bladder dysfunction in the IDO group $(71.96 \%$ female vs. $28.04 \%$ male patients), and statistically neurological injury shows demography of $59.5 \%$ women: $40.5 \%$ male. There was an improvement in the continence rate in both groups of patients. As $68.7 \%$ of IDO patients and $51.3 \%$ of NDO patients became dry after receiving their injection with BTXA. The percentage of dry patients among the IDO group was $68.7 \%(81 / 118)$ was higher when compared to patients suffering from NDO 51.3\% (20/39) (Figure 1).

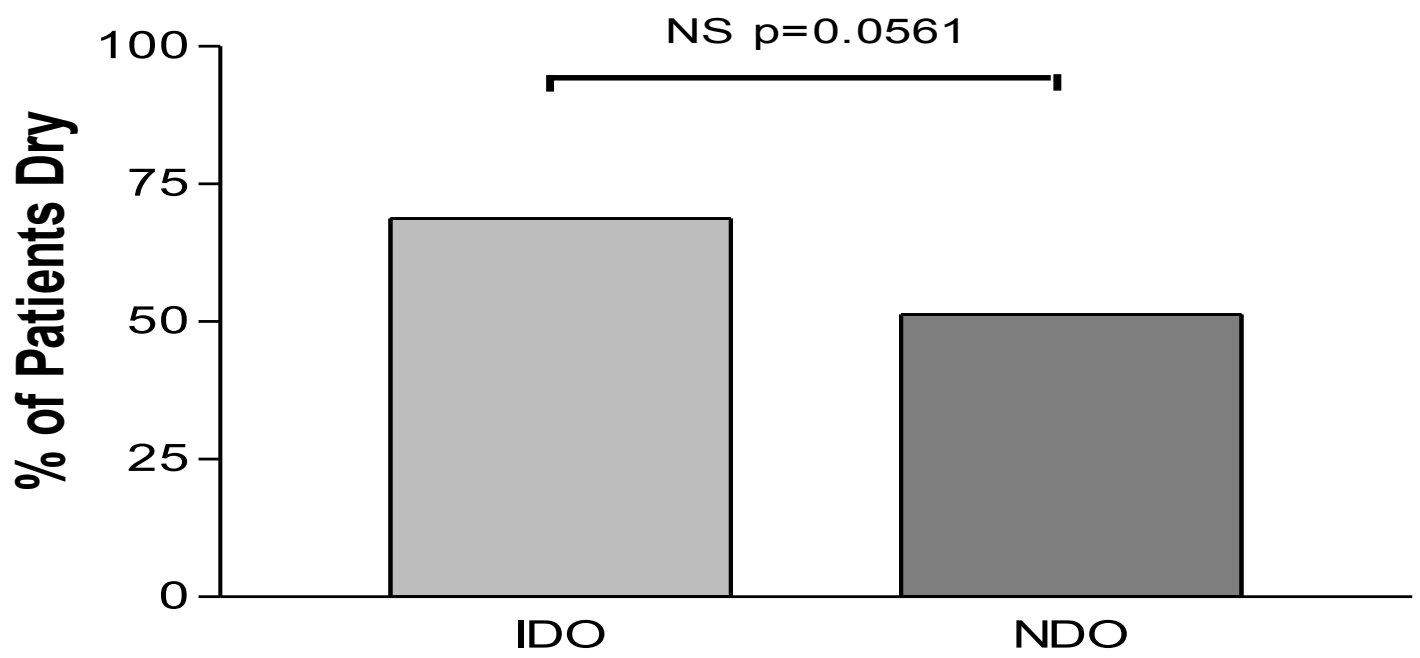

Figure 1: The percentage of Dry/Continent patients post Botox® $(I D O=$ Idiopathic Detrusor Over activity, $\mathrm{NDO}=$ Neurogenic Detrusor Over activity, $p=$ Probability value, NS= Not significant) 
The other variable which has been reported was patient's satisfaction with BTX-A treatment. Similar to dryness, there was a high number of patients $74.6 \%$ of IDO patients and $64.1 \%$ of NDO patients that were satisfied with the outcome of the BTX-A injection (Figure 2). Although some of the patients were still technically not dry, they had fewer symptoms, less pads being used, fewer ISC episodes, or urinary frequency rate. Comparison of satisfaction with the results and dryness was similar and comparable in the IDO group $74.6 \%$ satisfied and $68.7 \%$ dry. The NDO group was not so comparable $64.1 \%$ satisfied but $51.3 \%$ was dry. Information and advice was sought from the local statistician to establish a statistically significant cohort. The Fisher Exact Test was used to interpret the data as opposed to the Chi-square test as it was more accurate for this particular study. GraphPad Software 1994-1999 was used to analyse the data of dryness and satisfaction. The difference in Dry or Not Dry between Males and Females within the IDO, NDO groups, and male and females in those groups was analysed. The same steps were followed to interpret the Satisfied and Not Satisfied responses (Figure 4). When the Fisher Exact Test was applied, these results were not statistically significant $(p>0.5)$ in any group subjected to comparison. However, this does not indicate that there was no significant clinical improvement in continence and patient satisfaction rate. Nevertheless, when the dryness rate is compared between the two groups of patients (IDO and NDO) (Figure 1), it would need one extra patient to become dry to induce a $p$ value of less than 0.05 and make the result statistically significant. The incidence of UTIs in IDO patients is comparable to the published literature, ${ }^{19}$ but the NDO group showed less UTIs than the IDO group. $4.7 \%$ in NDO group compared to $6.06 \%$ in IDO group. This is probably due to the fact that patient with NDO are more prone to frequent infection and instrumentation which possibly increases the local immunity of the bladder to infection. The mean duration of action of BTX \pm standard deviation was $10.15( \pm 3.39)$ and $10.76( \pm 3.63)$ month in IDO and NDO respectively. This is comparable to the published literature. ${ }^{12,21}$ However, the mean duration of action between the second and third Botox $\AA$ injection \pm the standard deviation was 8.43 $( \pm 2.82)$ and $7.8( \pm 2.04)$ in IDO and NDO respectively, which is slightly shorter than the first injection. The issue of whether there is a difference between injection intervals or not is controversial.

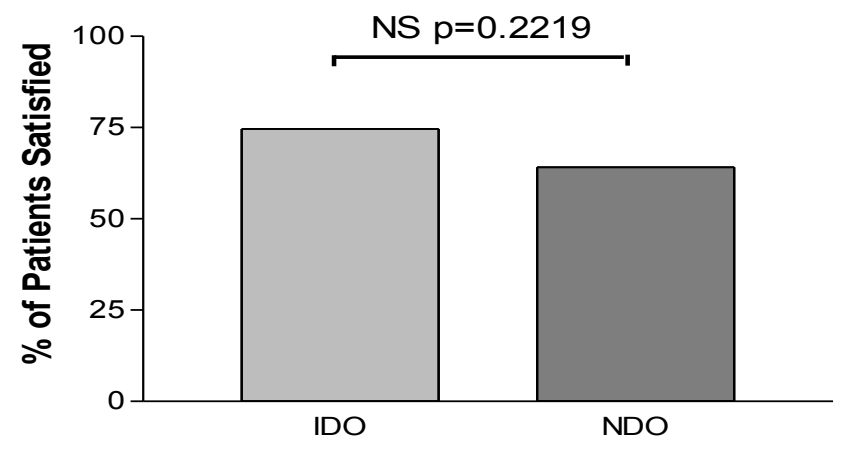

Figure 2: the percentage of patients satisfied with the Botox® injection(IDO= Idiopathic

Detrusor Over activity, NDO= Neurogenic Detrusor Over activity, $p=$ Probability value, NS= Not significant) 
Whereas 2 studies have showed repeated injection are associated with sustained beneficial effects 20,21 . Others report a shorter duration of action ofup to 5-6 months ${ }^{22,23}$. The reason for this wide range of reported duration is still unknown. However, the differences in the doses, injection technique, volume of diluent, number of injection sites and the diameter of the needle may be the cause ${ }^{22,23}$. Patients reported the duration of action of Botox ${ }^{\circledR}$ as 9-12 months with 4 patients reporting effectiveness of more than 12 month. The average duration of effectiveness

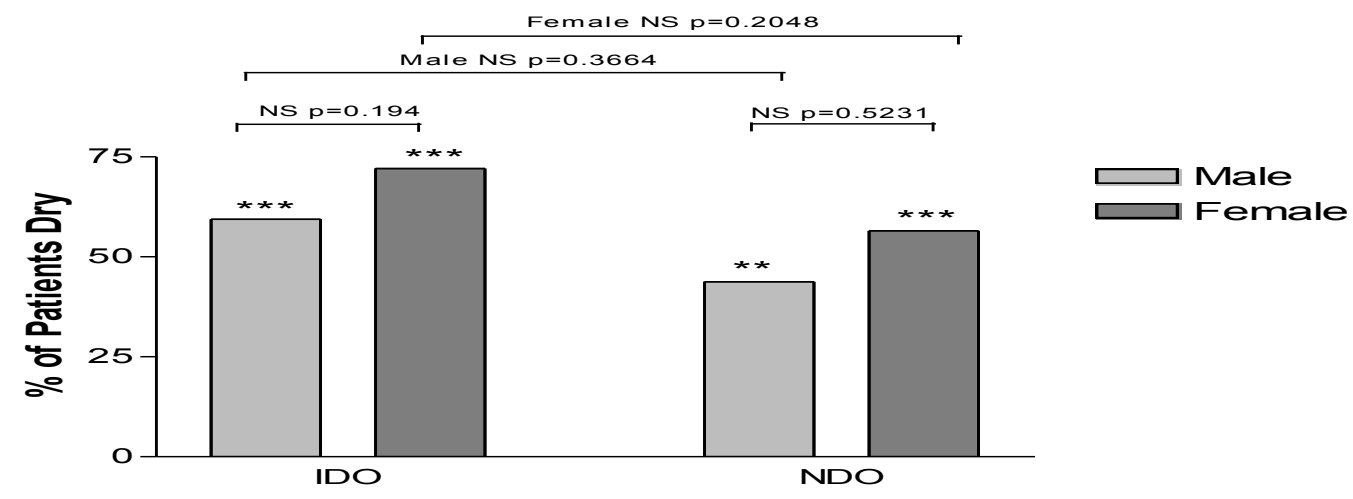

Figure 3: the percentage of patients Dry post Botox® injection(IDO= Idiopathic Detrusor Over activity, $\mathrm{NDO}=$ Neurogenic Detrusor Over activity, $p=$ Probability value, NS= Not significant, ${ }^{*}=$ Difference before and after BTX injection)

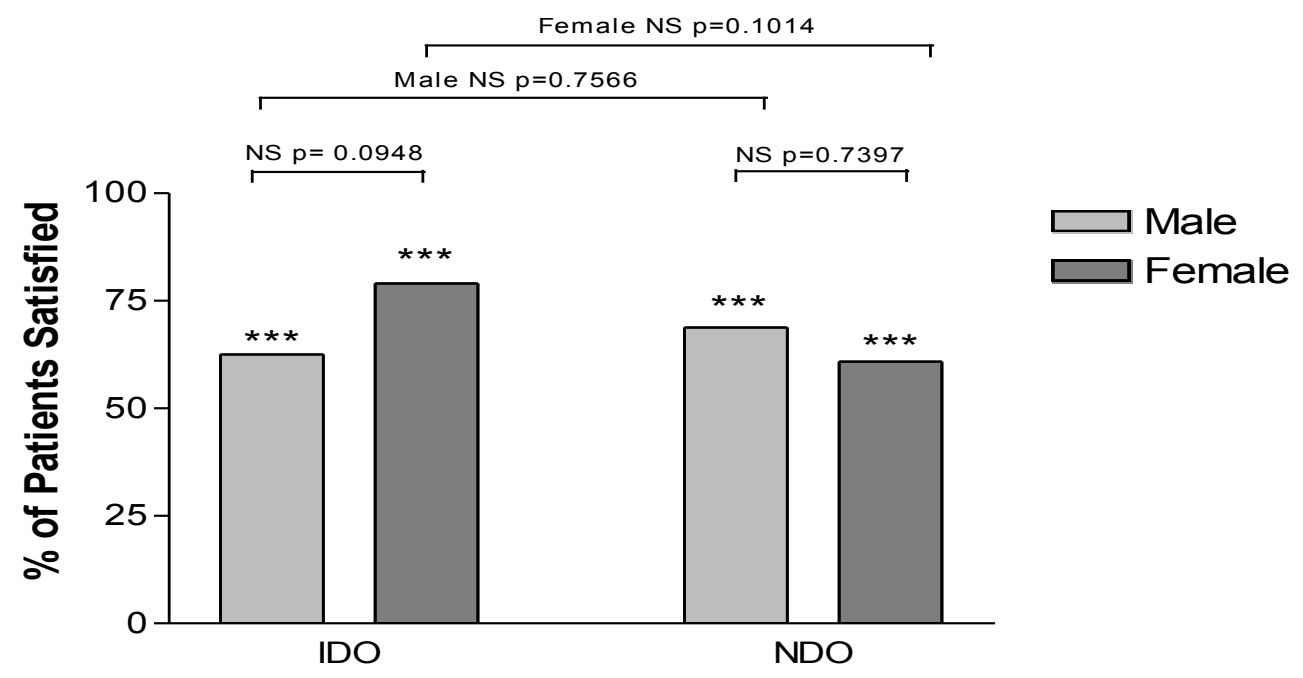

Figure 4: the percentage of patients Satisfied with Botox® injection(IDO= Idiopathic Detrusor Over activity, NDO= Neurogenic Detrusor Over activity, $p=$ Probability value, NS= Not significant, ${ }^{*}=$ Difference before and after BTX injection) 
effectiveness \pm standard deviation was $10.76( \pm 3.63)$ month. The mean duration of action between the second and third Botox ${ }^{\circledR}$ injection \pm the standard deviation was $7.8( \pm 2.04)$. The NDO group had one incidence of urinary retention in a group of 4 patients that were voiding pre Botox ${ }^{\circledR}$. A rate of $25 \%$ can be agreed as not representative for such a small group. Interestingly, one other person went on to have a suprapubic catheter inserted, but it is not clear if this was due to urinary retention or not from the note.

\section{Conclusion}

BTX-A treatment contributes to controlling quality of life symptoms and is not a long term cure. The IDO group responded better to Botox® treatment compared to the NDO group. It can be argued that this is because NDO patients invariably have Detrusor Sphincter Dyssynergia (DSD). This is because detrusor over activity drives sphincter dyssynergia resulting in very highpressures in the bladder and causes trabeculation. NDO patients require anticholinergics at doses higher than normal treatment and beyond the licensed recommendation. According to a new study by Silva et al ${ }^{24} 90 \%$ of patients were happy to consider continuing with BTX-A injections as a long term treatment option rather than having a big major operation. As far as the cost of treatment is concerned, one can argue using BTX-A is more cost effective than reconstructive surgery.

\section{References}

1.Greenwell, T.J., Venn, S.N., Mundy, A.R.: Augmentation cystoplasty. BJU International,2001, 88: 511-525.

2.Chartier-Castler, E., Igawa, Y., Kovindha, A., Radziszewski, P., Stone, A., Wiesel, P., Castro, D., Madersbacher, H.,Wyndaele, J.J.. Neurologic urinary and faecal incontinence. In: Incontinence, vol. 2. (eds). Paris: Health Publications Ltd. 2005,pp 1059-1162

3.Herbison, P.: Standards of efficacy for evaluation of treatment outcomes in urinary incontinence: recommendations of the Urodynamic Society.
Neurourology and Urodynamics, 1998,17: 153-154.

4. Hashim, H., Abrams, P. : Drug treatment of overactive bladder: efficacy, cost and quality-of-life considerations. Drugs,2004, 64: 1643-1656.

5. Khullar, V., Chapple, C., Gabriel, Z., Dooley, J.A. : The effects of antimuscarinics on health-related quality of life in overactive bladder: a systematic review and metaanalysis. Urology,2006, 68: 3848.

6. Abrams, P., Blaivas, J.G., Fowler, C.J., Fourcroy, J.L., Macdiarmid, S.A., Siegel, S.W., Van Kerrebroeck, $P$. : The role of neuromodulation in the management of urinary urge incontinence. BJU International ,2003,91: 355-359.

7. Herschorn, S., Hewitt, R.J. : Patient perspective of long-term outcome of augmentation cystoplasty for neurogenic bladder. Urology,1998, 52: 672-678.

8. Del Popolo, G., Filocamo, M.T., Li Marzi, V., Macchiarella, A., Cecconi, F., Lombardi, G., Nicita, G.: Neurogenic detrusor over activity treated with English botulinum toxin A: 8-year experience of one single centre. European Urology,2008, 53: 10131020.

9.Petersen, T., Nielsen, J.B., Schroder, H.D.: Intravesical capsaicin in patients with detrusor hyperreflexia - a placebo-controlled cross-over study. Scandinavian Journal of Urology and Nephrology ,1999,33: 104-110.

10. Giannantoni, A., Mearini, E., Del Zingaro, M., Porena M. : Six- Year Follow-Up of Botulinum Toxin A Intradetrusorial Injections in Patients with Refractory Neurogenic Detrusor Over activity: Clinical and Urodynamic Results. European Urology,2009, 55: 705-712.

11. Schulte-Baukloh, H., Weiss, C., Stolze, T., Herholz, J., Stürzebecher, B., Miller, K., Knispel, H.H: Botulinum-A toxin detrusor and sphincter injection in treatment of overactive bladder syndrome: objective outcome and patient satisfaction. European Urology 2005; 48: 984-990

12. Kuo, H.C.: Will suburothelial injection of small dose of botulinum A toxin have similar therapeutic effects and less adverse events for refractory detrusor over activity? Urology ,2006,68: 993-997.

13. Reitz, A., Denys, P., Fermanian, C., Schurch, B., Comperat, E., Kartier-Chastler, E. : Do repeat intradetrusor Botulinum toxin type a injections yield valuable results? Clinical and urodynamic results after five injections in patients with neurogenic detrusor over activity. European Urology,2007;52: 1729-1735.

14. Harper, M., Fowler, C.J., Dasgupta, P.: Botulinum toxin and its applications in the lower urinary tract. BJU International ,2004, 93:702-706.

15. Rajkumar, G.N., Small, D.R., Mustafa, A.W., Conn, G.: A prospective study to evaluate the safety, tolerability, efficacy and durability of response of intravesical injection of botulinum toxin type $A$ into detrusor muscle in patients with refractory idiopathic detrusor over activity. BJU 
International,2005, 96: 848-852.

16. Popat, R., Apostolidis, A., Kalsi, V., Gonzales, G., Fowler, C.J., Dasgupta, P. : A comparison between the response of patients with idiopathic detrusor over activity and neurogenic detrusor over activity to the first intradetrusor injection of botulinum-A toxin. The Journal of Urology ,2005,174: 984-989.

17. Leippold, T., Reitz, A. and Schurch, B. : Botulinum toxin as a new therapy option for voiding disorders: current state of the art. European Urology ,2003,44: 165.

18. Ruffion, A., Capelle, O., Paparel, P., Leriche, B., Leriche, A., Grise, P.: What is the optimum dose of type $A$ botulinum toxin for treating neurogenic bladder over activity? BJU International,2008, 97: 1030 -1034 .

19. Hori, S., Patki, P., Attar, K.H., Ismail, S., Vasconcelos, J.C., Shah, P.J.R.: Patients' perspective of botulinum toxin-A as a long-term treatment option for neurogenic detrusor over activity secondary to spinal cord injury. BJU International ,.2009,14641468.

20. Karsenty, G., Reitz, A., Lindemann, G., Boy, S., Schurch, B.: Persistence of therapeutic effect after repeated injections of botulinum toxin type $A$ to treat incontinence due to neurogenic detrusor over activity. Urology,2006, 68: 1193-1197.

21. De Seze, M., Wiart, L., Joseph, P.A., Dosque, J.P., Mazaux, J.M., Barat, M.: Capsaicin and neurogenic detrusor hyperreflexia: a double-blind placebo-controlled study in 20 patients with spinal cord lesions. Neurourology and Urodynamics ,1998,17: 513-523.

22.Kalsi,V., Popat, R.B., Apostolidis, A., Kavia, R., Odeyemi, I.A., Dakin, H.A.,Warner, J., Elneil, S., Fowler, C.J., Dasgupta, P.: Cost-consequence analysis evaluating the use of botulinum neurotoxin -A in patients with detrusor over activity based on clinical outcomes obseved at a single UK centre. European Urology 2006b; 49: 519-527.

23. Kopp, Z., Irwin, D.E., Agatep, B., Milsom, I., Abrams, P. . Worldwide Estimates of Current and Future Individuals ( $\geq 20$ years) With Lower Urinary Tract Symptoms Including Urinary Incontinence and Overactive Bladder. Poster presentation, 4th International Consultation on Incontinence, Paris.2008.

24. Silva, C., Silva, J., Ribeiro, M.J., Avelino, A., Cruz, F.: Urodynamic effect of intravesical resiniferatoxin in patients with neurogenic detrusor over activity of spinal origin: results of a double-blind randomized placebo-controlled trial. European Urology ,2005,48: 650-655. 